\title{
A Second-Order Cone Programming Approach for Minimax Design of 2-D FIR Filters with Low Group Delay
}

\author{
Wu-Sheng Lu \\ Dept. of Electrical and Computer Engineering \\ University of Victoria \\ Victoria, BC, Canada V8@ 3P6 \\ Email: wslu@ece.uvic.ca
}

\author{
Takao Hinamoto \\ Graduate School of Engineering \\ Hiroshima University \\ Higashi-Hiroshima, 739-8527, Japan \\ Email: hinamoto@hiroshima-u.ac.jp
}

\begin{abstract}
A design algorithm based on second-order cone programming (SOCP) for minimax design of 2-D FIR filters with low group delay is proposed. SOCP is a special class of convex programming problems that can be carried out considerably more efficiently than the popular semidefinite programming. The simulation studies presented in this paper also confirm this in a filter design context. The proposed algorithm is compared favorably with a recently proposed design method based on sequential quadratic programming. The proposed design method is expected to be a useful utility for 2-D digital filter designers whose interest is not limited to linear phase responses and low order filters.
\end{abstract}

\section{INTRODUCTION}

There have been several algorithms for the design of twodimensional (2-D) digital filters, see for example [1]-[7] and the reference cited therein. Concerning the minimax designs, a successful early attempt is documented in [1]. More recent efforts include [6] and [7] where methods based on semidefinite programming (SDP) and sequential quadratic programming (SQP) have been proposed. These methods work quite well except that the design complexity becomes rather high even for filters of moderate order. The high computational complexity is attributed to two facts: the large number of design variables in the order of $O\left(N 1 \cdot N_{2}\right)$ for filters of size $N_{1} \times N_{2}$ and the large number of constraints imposed on a dense set of frequency grid points over the baseband $[-\pi, \pi] \times[-\pi, \pi]$.

In this paper, the minimax design of 2-D FIR filters approximating arbitrary frequency responses (including those with low group delays), is addressed in a convex programming framework known as second-order cone programming (SOCP) [8]. The paper provides details in design formulation and algorithmic description of the method, and presents simulation results to demonstrate that the SOCP-based algorithm can achieve the same design results with much reduced computational complexity relative to the algorithms in the literature.

\section{Problem Formulation}

Consider a 2-D FIR transfer function

$$
H\left(z_{1}, z_{2}\right)=\sum_{i=0}^{N_{1}-1} \sum_{j=0}^{N_{2}-1} h_{i j} z_{1}^{-i} z_{2}^{-j}
$$

We seek to find a transfer function $H\left(z_{1}, z_{2}\right)$ in (1) whose frequency response best approximates a desired frequency response $H_{d}\left(\omega_{1}, \omega_{2}\right)$ in the minimax sense. Namely, we are interested in solving the optimization problem

$$
\underset{\boldsymbol{h}}{\operatorname{minimize}} \underset{\left(\omega_{1}, \omega_{2}\right) \in \Omega}{\operatorname{maximize}} W\left(\omega_{1}, \omega_{2}\right)\left|H\left(\omega_{1}, \omega_{2}\right)-H_{d}\left(\omega_{1}, \omega_{2}\right)\right|
$$

where $\Omega=\left\{\left(\omega_{1}, \omega_{2}\right),-\pi \leq \omega_{1}, \omega_{2} \leq \pi\right\}$ and $W\left(\omega_{1}, \omega_{2}\right) \geq$ 0 is a known weighting function defined on $\Omega$.

For notation simplicity, we assume $N_{1}=N_{2}=N$ and denote

$$
\begin{aligned}
H\left(\omega_{1}, \omega_{2}\right) & =H\left(e^{j \omega_{1}}, e^{j \omega_{2}}\right) \\
\boldsymbol{c}(\omega) & =\left[\begin{array}{lll}
1 & \cos \omega & \cdots \\
\boldsymbol{s}(\omega) & =\left[\begin{array}{lll}
0 & \sin \omega & \cos (N-1) \omega
\end{array}\right]^{T} & \sin (N-1) \omega
\end{array}\right]^{T}
\end{aligned}
$$

and

$$
\boldsymbol{H}=\left\{h_{i j}, 0 \leq i, j \leq N-1\right\}
$$

The frequency response of the 2-D FIR filter characterized in (1) can be expressed as

$$
\begin{aligned}
H\left(\omega_{1}, \omega_{2}\right)= & {\left[\boldsymbol{c}^{T}\left(\omega_{1}\right) \boldsymbol{H} \boldsymbol{c}\left(\omega_{2}\right)-\boldsymbol{s}^{T}\left(\omega_{1}\right) \boldsymbol{H} \boldsymbol{s}\left(\omega_{2}\right)\right] } \\
& -j\left[\boldsymbol{s}^{T}\left(\omega_{1}\right) \boldsymbol{H} \boldsymbol{c}\left(\omega_{2}\right)+\boldsymbol{c}^{T}\left(\omega_{1}\right) \boldsymbol{H} \boldsymbol{s}\left(\omega_{2}\right)\right]
\end{aligned}
$$

If we let $\boldsymbol{c}_{i}=\boldsymbol{c}\left(\omega_{i}\right)$ and $\boldsymbol{s}_{i}=\boldsymbol{s}\left(\omega_{i}\right)$ for $i=1,2$, then we can write

$$
\begin{aligned}
& H\left(\omega_{1}, \omega_{2}\right)=\operatorname{tr}\left[\boldsymbol{P}\left(\omega_{1}, \omega_{2}\right) \boldsymbol{H}\right]-j \operatorname{tr}\left[\boldsymbol{Q}\left(\omega_{1}, \omega_{2}\right) \boldsymbol{H}\right] \\
& \boldsymbol{P}\left(\omega_{1}, \omega_{2}\right)=\boldsymbol{c}_{2} \boldsymbol{c}_{1}^{T}-\boldsymbol{s}_{2} \boldsymbol{s}_{1}^{T} \\
& \boldsymbol{Q}\left(\omega_{1}, \omega_{2}\right)=\boldsymbol{c}_{2} \boldsymbol{s}_{1}^{T}+\boldsymbol{s}_{2} \boldsymbol{c}_{1}^{T}
\end{aligned}
$$

where $\operatorname{tr}[\cdot]$ denotes matrix trace. Further, if we denote the column vectors generated by stacking the transposed rows of $\boldsymbol{P}\left(\omega_{1}, \omega_{2}\right), \boldsymbol{Q}\left(\omega_{1}, \omega_{2}\right)$ and $\boldsymbol{H}$ by $\boldsymbol{p}\left(\omega_{1}, \omega_{2}\right), \boldsymbol{q}\left(\omega_{1}, \omega_{2}\right)$ and 
$\boldsymbol{h}$, respectively, then the frequency response can simply be expressed as

$$
H\left(\omega_{1}, \omega_{2}\right)=\boldsymbol{p}^{T}\left(\omega_{1}, \omega_{2}\right) \boldsymbol{h}-j \boldsymbol{q}^{T}\left(\omega_{1}, \omega_{2}\right) \boldsymbol{h}
$$

By placing an upper bound $\eta$ on the objective function in (2), the solution of (2) shall minimize the bound, thus the problem at hand can be converted to

minimize $\quad \eta$

subject to: $W\left(\omega_{1}, \omega_{2}\right)\left\{\left[\boldsymbol{p}^{T}\left(\omega_{1}, \omega_{2}\right) \boldsymbol{h}-H_{d r}\left(\omega_{1}, \omega_{2}\right)\right]^{2}\right.$

$$
\left.+\left[\boldsymbol{q}^{T}\left(\omega_{1}, \omega_{2}\right) \boldsymbol{h}-H_{d i}\left(\omega_{1}, \omega_{2}\right)\right]^{2}\right\}^{1 / 2} \leq \eta(4 \mathrm{~b})
$$

$$
\text { for }\left(\omega_{1}, \omega_{2}\right) \in \Omega
$$

where $H_{d r}\left(\omega_{1}, \omega_{2}\right)$ and $-H_{d i}\left(\omega_{1}, \omega_{2}\right)$ are the real and imaginary parts of $H_{d}\left(\omega_{1}, \omega_{2}\right)$, i.e.,

$$
H_{d}\left(\omega_{1}, \omega_{2}\right)=H_{d r}\left(\omega_{1}, \omega_{2}\right)-j H_{d i}\left(\omega_{1}, \omega_{2}\right)
$$

For the design of low group delay filters, the desired frequency response assumes the form

$$
H_{d}\left(\omega_{1}, \omega_{2}\right)=A_{d}\left(\omega_{1}, \omega_{2}\right) e^{-j d\left(\omega_{1}+\omega_{2}\right)}
$$

where $A_{d}\left(\omega_{1}, \omega_{2}\right)$ is a nonnegative real-valued function on $\Omega$, and $d$ is a constant group delay between 0 and $(N-1) / 2$. In this case we have

and

$$
H_{d r}\left(\omega_{1}, \omega_{2}\right)=A_{d}\left(\omega_{1}, \omega_{2}\right) \cos \left[d\left(\omega_{1}+\omega_{2}\right)\right]
$$

$$
H_{d i}\left(\omega_{1}, \omega_{2}\right)=A_{d}\left(\omega_{1}, \omega_{2}\right) \sin \left[d\left(\omega_{1}+\omega_{2}\right)\right]
$$

It should be stressed, however, that both the problem formulation in (4) and the solution process to be described below are applicable to the design of 2-D FIR filters approximating a given arbitrary frequency response.

For a feasible exercise of SOCP, the constraints in (4b) are discretized to a dense grid of frequencies $\Omega_{d}=$ $\left\{\left(\omega_{1 k}, \omega_{2 k}\right), k=1,2, \ldots, K\right\} \subseteq \Omega$ and, in doing so, the problem in (4) becomes

$$
\text { minimize } \boldsymbol{e}^{T} \boldsymbol{x}
$$

subject to: $\quad a_{k}(\boldsymbol{h}) \leq \boldsymbol{e}^{T} \boldsymbol{x} \quad$ for $k=1,2, \ldots, K(5 \mathrm{~b})$

where $\boldsymbol{x}=\left[\begin{array}{ll}\eta & \boldsymbol{h}^{T}\end{array}\right]^{T}, \boldsymbol{e}=\left[\begin{array}{llll}1 & 0 & \cdots & 0\end{array}\right]^{T}$, and

$$
a_{k}(\boldsymbol{h})=W_{k}\left[\left(\boldsymbol{p}_{k}^{T} \boldsymbol{h}-H_{d r k}\right)^{2}+\left[\boldsymbol{q}_{k}^{T} \boldsymbol{h}-H_{d i k}\right)^{2}\right]^{1 / 2}
$$

with

$$
\begin{aligned}
W_{k} & =W\left(\omega_{1 k}, \omega_{2 k}\right) \\
\boldsymbol{p}_{k} & =\boldsymbol{p}\left(\omega_{1 k}, \omega_{2 k}\right) \\
\boldsymbol{q}_{k} & =\boldsymbol{q}\left(\omega_{1 k}, \omega_{2 k}\right) \\
H_{d r k} & =H_{d r}\left(\omega_{1 k}, \omega_{2 k}\right) \\
H_{d i k} & =H_{d i}\left(\omega_{1 k}, \omega_{2 k}\right)
\end{aligned}
$$

\section{An SOCP-BASED SOLUTION}

\section{A. $S O C P$}

SOCP is a class of convex programming problems, in which a linear objective function is minimized subject to secondorder cone constraints [8]. Also known as quadratic or Lorentz cone, a second-order cone of dimension $n$ is defined as

$$
\mathcal{K}=\left\{\left[\begin{array}{c}
t \\
\boldsymbol{u}
\end{array}\right], t \in R, \boldsymbol{u} \in R^{n-1},\|\boldsymbol{u}\|_{2} \leq t\right\}
$$

For $n=1$, the second-order cone is degenerated to a ray on the $t$-axis starting from $t=0$ and for $n=3$ the second-order cone is depicted in Fig. 1.

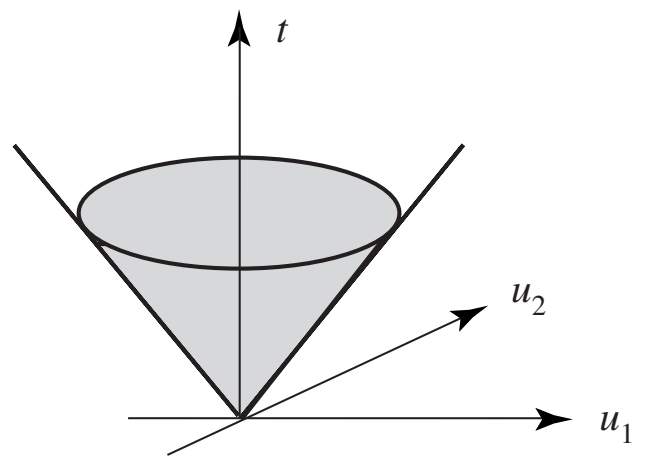

Fig. 1. Second-order cone of dimension $n=3$.

A standard SOCP formulation favored in many engineering applications can be expressed as

$$
\begin{aligned}
\operatorname{minimize} & \boldsymbol{b}^{T} \boldsymbol{x} \\
\text { subject to: } & \left\|\boldsymbol{A}_{k}^{T} \boldsymbol{x}+\boldsymbol{c}_{k}\right\|_{2} \leq \boldsymbol{b}_{k}^{T} \boldsymbol{x}+d_{k} \\
& \text { for } k=1,2, \ldots, K
\end{aligned}
$$

The reason we call (6) an SOCP problem is because of the fact that if we let

$$
\hat{\boldsymbol{A}}_{k}^{T}=\left[\begin{array}{c}
\boldsymbol{b}_{k}^{T} \\
\boldsymbol{A}_{k}^{T}
\end{array}\right], \hat{\boldsymbol{c}}_{k}=\left[\begin{array}{l}
d_{k} \\
\boldsymbol{c}_{k}
\end{array}\right]
$$

then the constraints in (6b) are equivalent to that each $\hat{\boldsymbol{A}}_{k}^{T} \boldsymbol{x}+$ $\hat{\boldsymbol{c}}_{k}$ belongs to a second-order cone $\mathcal{K}_{k}$.

\section{B. An SOCP formulation of problem (5)}

The function $a_{k}(\boldsymbol{h})$ in $(5 \mathrm{c})$ can be written as

$$
\begin{aligned}
a_{k}(\boldsymbol{h})= & {\left[\left(\hat{\boldsymbol{p}}_{k}^{T} \boldsymbol{h}-\hat{H}_{d r k}\right)^{2}+\left(\hat{\boldsymbol{q}}_{k}^{T} \boldsymbol{h}-\hat{H}_{d i k}\right)^{2}\right]^{1 / 2} } \\
& =\left\|\left[\begin{array}{c}
\hat{\boldsymbol{p}}_{k}^{T} \\
\hat{\boldsymbol{q}}_{k}^{T}
\end{array}\right] \boldsymbol{h}-\left[\begin{array}{c}
\hat{H}_{d r k} \\
\hat{H}_{d i k}
\end{array}\right]\right\|_{2} \\
& =\left\|\left[\begin{array}{cc}
0 & \hat{\boldsymbol{p}}_{k}^{T} \\
0 & \hat{\boldsymbol{q}}_{k}^{T}
\end{array}\right] \boldsymbol{x}+\left[\begin{array}{c}
\hat{H}_{d r k} \\
\hat{H}_{d i k}
\end{array}\right]\right\|_{2}
\end{aligned}
$$


where $\hat{\boldsymbol{p}}_{k}=W_{k}^{1 / 2} \boldsymbol{p}_{k}, \hat{\boldsymbol{q}}_{k}=W_{k}^{1 / 2} \boldsymbol{q}_{k}, \hat{H}_{d r k}=W_{k}^{1 / 2} H_{d r k}$, and $\hat{H}_{d i k}=W_{k}^{1 / 2} H_{d i k}$. Hence the constraints in (5b) become the constraints in $(6 \mathrm{~b})$ with

$$
\boldsymbol{A}_{k}=\left[\begin{array}{cc}
0 & 0 \\
\hat{\boldsymbol{p}}_{k} & \hat{\boldsymbol{q}}_{k}
\end{array}\right], \boldsymbol{b}_{k}=\boldsymbol{e}, \boldsymbol{c}_{k}=-\left[\begin{array}{c}
\hat{H}_{d r k} \\
\hat{H}_{d i k}
\end{array}\right], d_{k}=0
$$

and the objective function in (5a) becomes the objective functin in (6a) with $b=e$.

\section{MATLAB implementation}

MATLAB toolbox SeDuMi version $1.1 \mathrm{R} 2$ was used to solve the SOCP problem in (6) where the data required are specified in (7). The name of the toolbox stands for selfdual minimization as it implements a self-dual embedding technique for optimization over self-dual homogeneous cones [9].

For implementation purposes, define

$$
\begin{aligned}
& \boldsymbol{A}_{t}=\left[\begin{array}{llll}
\tilde{\boldsymbol{A}}_{1} & \tilde{\boldsymbol{A}}_{2} & \cdots & \tilde{\boldsymbol{A}}_{K}
\end{array}\right] \\
& \tilde{\boldsymbol{A}}_{k}=-\left[\begin{array}{ll}
\boldsymbol{b}_{k} & \boldsymbol{A}_{k}
\end{array}\right] \\
& \boldsymbol{b}_{t}=-\boldsymbol{b} \\
& \boldsymbol{c}_{t}=\left[\begin{array}{c}
\tilde{\boldsymbol{c}}_{1} \\
\vdots \\
\tilde{\boldsymbol{c}}_{K}
\end{array}\right], \tilde{\boldsymbol{c}}_{k}=\left[\begin{array}{c}
d_{k} \\
\boldsymbol{c}_{k}
\end{array}\right]
\end{aligned}
$$

In addition, define a $K$-dimensional vector $\boldsymbol{q}=$ $\left[\begin{array}{llll}n_{1} & n_{2} & \ldots & n_{K}\end{array}\right]$ where $n_{k}$ denotes the number of columns of matrix $\tilde{\boldsymbol{A}}_{k}$. The key commands for SeDuMi to solve problem (6) are as follows:

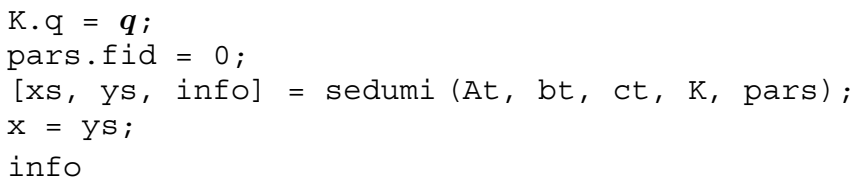

The solution of the problem is then given as vector $x$.

\section{Design EXAMPles}

The proposed algorithm was applied to design 2-D FIR filters of various types and sizes. As a representative, Fig. 2 depicts the amplitude response and passband group delay of a circularly symmetric lowpass filter of size $43 \times 43$ with normalized passband edge $\omega_{p}=0.5$ and stopband edge $\omega_{1}=$ 0.65 that approximates a desired lowpass frequency response with passband group delay $=19$. A total of $K=1986$ grid points. It took the proposed algorithm 25 iterations and 652.69 seconds CPU time on a $3.4 \mathrm{GHz}$ Pentium 4 PC to converge to the solution as shown in Fig. 2 whose maximum passband error and minimum stopband attenuation were found to be 0.0021 and $54.1529 \mathrm{~dB}$, respectively. The maximum relative deviation of group delay in passband was 0.0279 .

For comparison purposes, the proposed algorithm was also applied to design five linear-phase circularly symmetric lowpass filters with $f_{p}=0.425, f_{a}=0.575$, and $N=7,11,15$, 19 , and 23. The filters with the same design specifications were also carried out using the algorithms of [3] and [6] and the

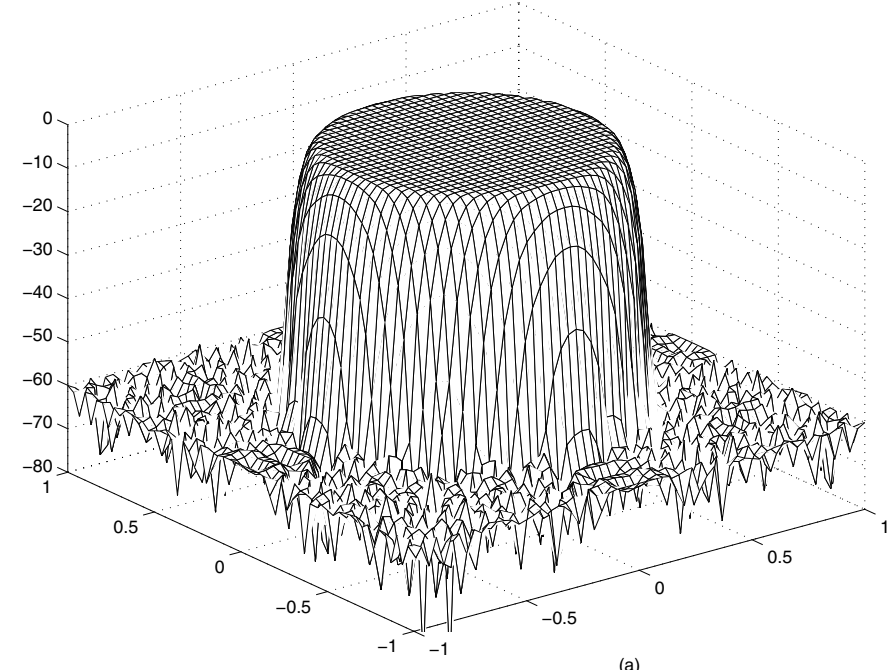

(a)

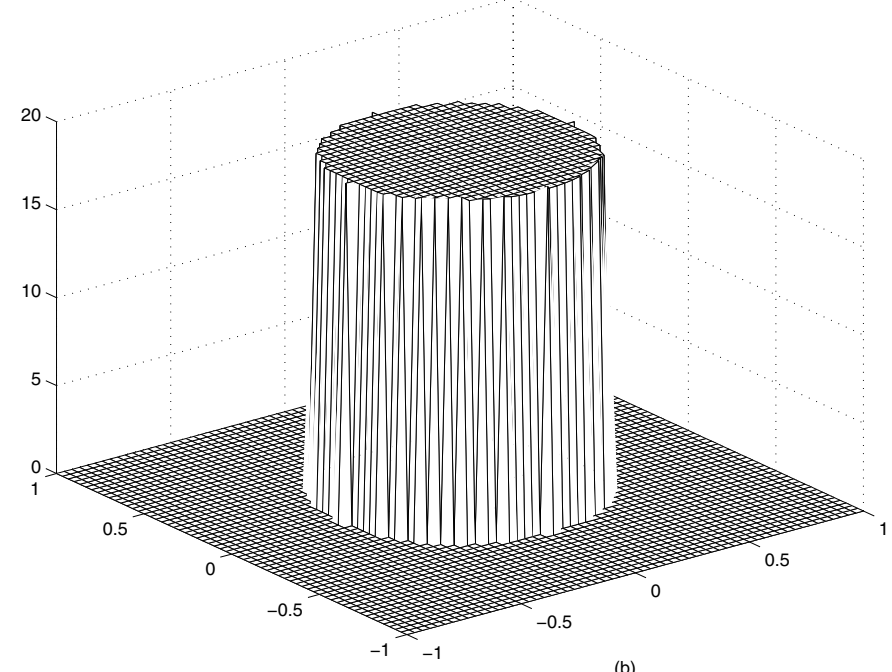

(b)

Fig. 2. (a) Amplitude response and (b) passband group delay of the $43 \times 43$ circularly symmetric lowpass filter.

results are summarized in Table 1. The amplitude responses of these filters are shown in Fig. 3. From these design examples, it is quite obvious that the SOCP-based algorithm can be used to design filters of fairly high order and offers considerably reduced computational complexity relative to that of several existing design methods.

\section{CONCLUSIONS}

The minimax design of 2-D filters with low group delay can be formulated as an SOCP problem which is a convex constrained optimization that admits a unique, global solution. Thanks to the public-domain toolbox SeDuMi which has proven to be a reliable and efficient LP-SDP-SOCP solver, the proposed design method is expected to be a useful utility for 2-D digital filter designers whose interest is not limited to linear phase responses and low order filters. 
TABLE I

COMPARISON OF THE PROPOSED METHOD WITH THE METHODS IN [3], [6]

\begin{tabular}{c|c|c|c|c|c|c}
\hline$N$ & Grid points & \multicolumn{2}{|c|}{ maximum ripple } & \multicolumn{3}{c}{ CPU time in seconds } \\
\cline { 3 - 7 } & $K$ & passband & stopband & $\begin{array}{c}\text { proposed } \\
\text { method }\end{array}$ & {$[3]$} & {$[6]$} \\
\hline 7 & 345 & 0.1769 & 0.1787 & 0.36 & 8.10 & 1.72 \\
\hline 11 & 345 & 0.0931 & 0.1093 & 0.83 & 15.74 & 3.36 \\
\hline 15 & 351 & 0.0629 & 0.0643 & 1.88 & 33.67 & 6.65 \\
\hline 19 & 629 & 0.0317 & 0.0451 & 8.71 & 117.99 & 23.42 \\
\hline 23 & 761 & 0.0193 & 0.0227 & 20.02 & 282.18 & 55.44 \\
\hline
\end{tabular}

\section{ACKNOWLEDGEMENT}

The first author is thankful to the Natural Sciences and Engineering Research Council of Canada for supporting this work.

\section{REFERENCES}

[1] J. H. McClellan and T. W. Parks, "Equiripple approximation of fan filters," Geophysics, vol. 7, pp. 573-583, 1972.

[2] Y. Kamp and J. P. Thiran, "Chebyshev approximation for two-dimensional nonrecursive digital filters," IEEE Trans. Circuits Syst., vol. 22, pp. 208 218, March 1975.

[3] C. Charalambous, "The performance of an algorithm of minimax design of two-dimensional linear phase FIR figital filters," IEEE Trans. Circuits Syst., vol. 32, pp. 1016-1028, Oct. 1985.

[4] D. T. Nguyen and M. N. S. Swamy, "Approximation design of 2-D digital filters with elliptical magnitude response of arbitrary orientation," IEEE Trans. Circuits Syst., vol. 33, pp. 597-603, June 1986.

[5] W.-P. Zhu, M. O. Ahmad, and M. N. S. Swamy, "A closed-form solution to the least-square design problem of 2-D linear-phase FIR filters," IEEE Trans. Circuits Syst., II, vol. 44, pp. 1032-1039, Dec. 1997.

[6] W.-S. Lu, "A unified approach for the design of 2-D digital filters via semidefinite programming," IEEE Trans. Circuits Syst., vol. 49, pp. 814826, June 2002.

[7] W.-S. Lu and T. Hinamoto,"A new minimax design for 2-D FIR filters with low group delay," ISCAS’2005, pp. 2028-2031, Kobe, Japan, May 2005.

[8] M. S. Lobo, L. Vandenberghe, S. Boyd, and H. Lebret, "Applications of second-order cone programming," Linear Algebra and Applications, vol. 248, pp. 193-228, Nov. 1998

[9] J. F. Sturm, "Using SeDuMi 1.02, a MATLAB toolbox for optimization over symmetric cones," Optimization Methods and Software, vol. 1112, pp. 625-653, 1999. (Version 1.1R2 of SeDuMi is available at http://sedumi.mcmaster.ca/)
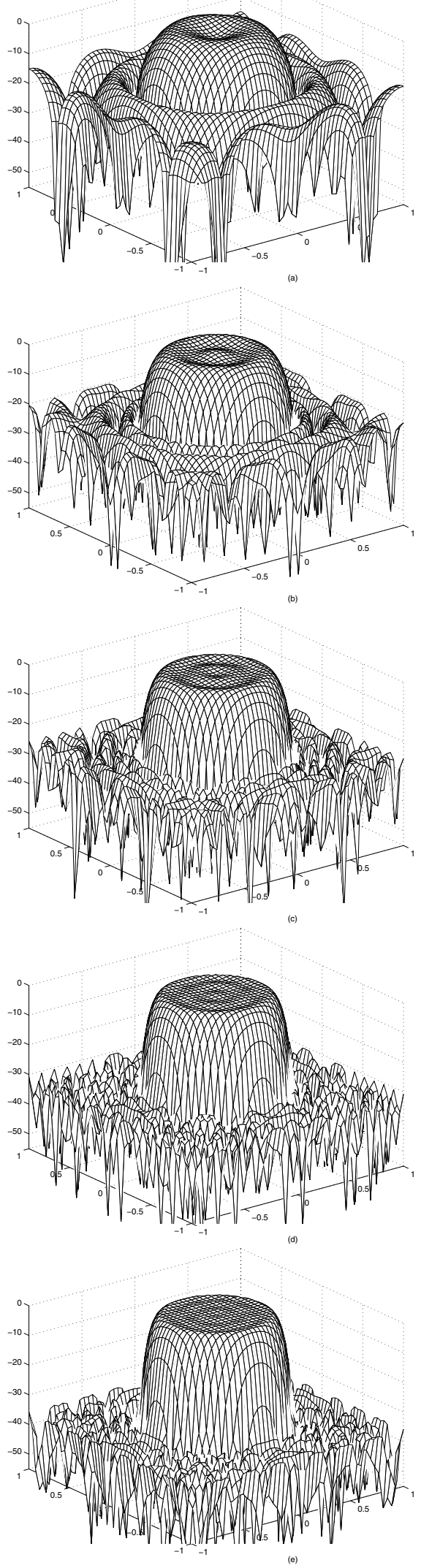

Fig. 3. Amplitudes of circularly symmetric linear-phase lowpass 2-D FIR filters of size (a) $7 \times 7$, (b) $11 \times 11$, (c) $15 \times 15$, (d) $19 \times 19$, and (e) $23 \times 23$. 